\title{
Uveal metastatic carcinoma in human immunodeficiency virus infection
}

PS Riske1, Jl Perlman 1,2,3,4, JJ Moy4, JS Ohr², MD Raible ${ }^{2}$, R Weiss $^{4}$ and MJ Daily ${ }^{1}$

Abstract

We report the occurrence of uveal metastatic carcinoma in two patients with longstanding HIV infection presenting with decreased visual acuity. In the first case, a 49-year-old man with a 6-year history of HIV infection presented with a 4-5 month history of blurred vision in his right eye. In the second case, a 53-year-old man with a 5-year history of HIV infection presented with a 3-week history of distorted and blurred vision in both eyes. In both cases, a choroidal metastatic carcinoma was ultimately discovered. To our knowledge, these are the first reported cases of metastatic uveal carcinoma in individuals with HIV infection. Currently, there have been dramatic improvements in treatment for HIV infection and longer survival times of infected individuals. This fact, together with reported increased frequencies and aggressiveness of carcinomas in HIV-infected individuals will likely result in increasing occurrences of uveal metastases from primary carcinomas in HIV.

Eye (2002) 16, 633-637. doi:10.1038/

sj.eye. 6700126

Keywords: metastatic carcinoma; HIV; AIDS; choroid; uvea; lung carcinoma

Metastatic tumors to the uvea are considered the most common intraocular tumors of adults. ${ }^{1}$ Primary tumors from breast and lung account for the majority of cases, with lung being the most common site of origin in men. ${ }^{2}$ Despite the increased incidence of malignancy in patients immunocompromised secondary to Human Immunodeficiency Virus (HIV), there are no reports in the literature of uveal metastases from malignant carcinoma in HIV. This is surprising given the reported increased incidence of lung carcinoma in patients with
HIV infection. ${ }^{3,4}$ We report two cases of metastatic choroidal carcinoma in patients with HIV infection.

\section{Case reports}

\section{Case 1}

A 49-year-old African-American man with a 6year history of HIV infection presented with a complaint of blurred vision in the right eye for 4-5 months. Prior medical history was also significant for diabetes mellitus, intravenous drug use, and heavy alcohol and tobacco use. The patient had a CD4 count of 452 , but was reported to be otherwise without evidence of immunocompromised related disease. The patient was initially evaluated by an optometrist with a diagnosis of a retinal detachment of the right eye. The patient was referred to an ophthalmologist who diagnosed a retinal detachment secondary to a choroidal melanotic mass. Visual acuity was count fingers at 6 inches in the right eye and 20/30 in the left eye. A diagnostic ultrasound was performed. A B-scan revealed a large nonmobile choroidal mass without evidence of internal vascularity or extrascleral extension (Figure 1a). The tumor measured $17.8 \times 16 \mathrm{~mm}$ with a height of $9.9 \mathrm{~mm}$. The findings were felt to be most consistent with a necrotic choroidal melanoma with atypical ultrasonographic findings (eg, low angle kappa). A metastatic tumor could not be ruled out, however, and the patient was referred to an oncologist for metastatic workup. An enucleation was advised. After being lost to follow-up for several months, the patient subsequently underwent an uneventful enucleation.

Evaluation by the oncologist included negative carcinoembryonic antigen and prostate specific antigen. Elevated liver enzymes were noted. Additional testing included left supraclavicular lymph node
${ }^{1}$ Department of Ophthalmology Loyola University Chicago Maywood, IL, USA

2Department of Pathology Loyola University Chicago Maywood, IL, USA

${ }^{3}$ Ophthalmology Section Surgical Service

Edward Hines Jr VA Hospital

Hines, IL, USA

${ }^{4}$ Department of Ophthalmology and Visual Sciences University of Illinois Chicago, IL, USA

Correspondence: Jl Perlman MD, PhD Loyola University Chicago Department of Ophthalmology 2160 South First Avenue Maywood, IL 60153, USA

Tel: 7082025134

Fax: 7082022180

E-mail:.jperlma@lumc.edu 

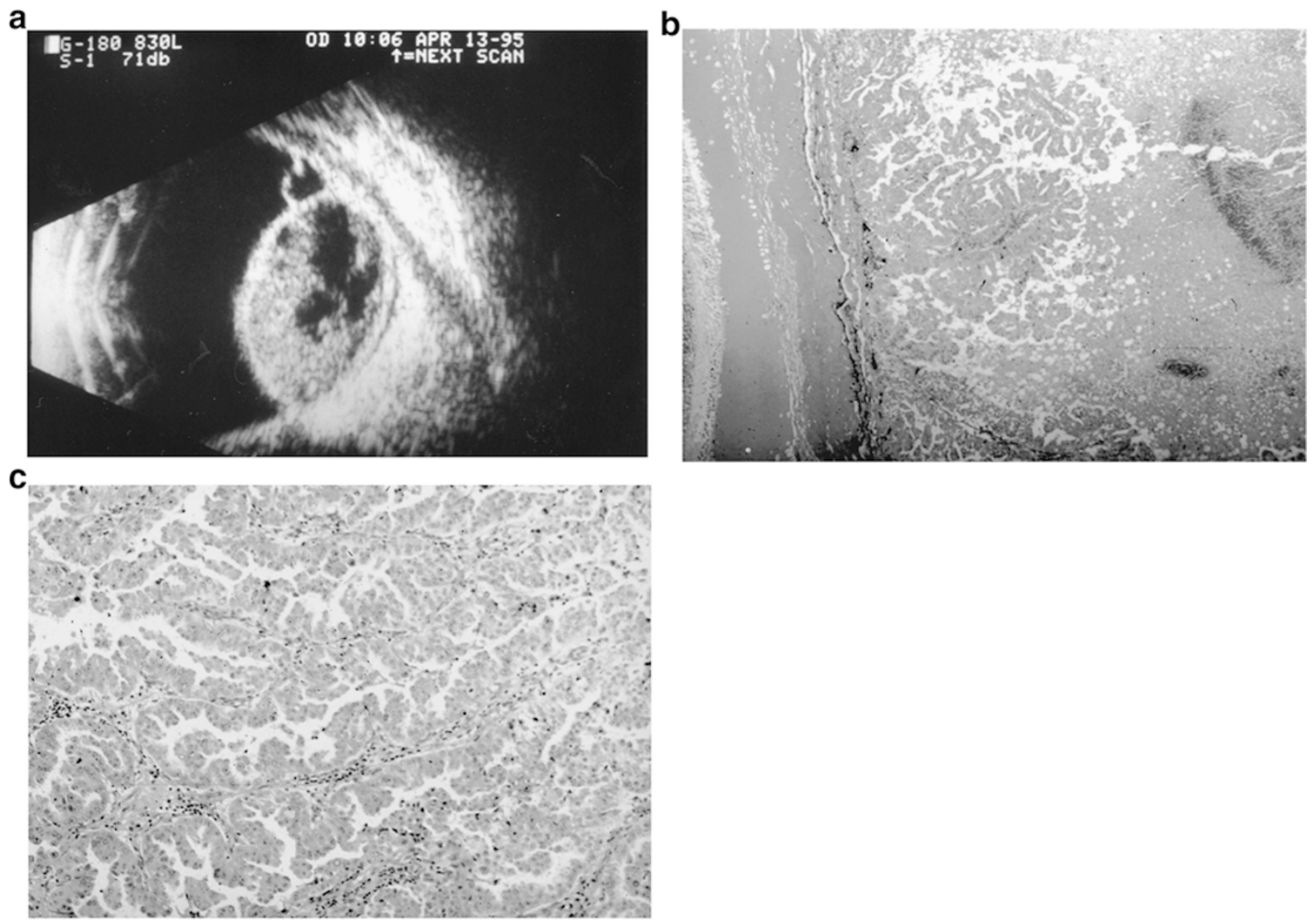

Figure 1 Case 1. (a) B-scan ultrasonography of right eye demonstrates sub-retinal choroidal mass. (b) Histologic section of the right eye shows sub-retinal adenocarcinoma with papillary configuration of mass. Note subretinal exudate at left (hematoxylin and eosin $\times 100$ ). (c) Histologic section from supraclavicular lymph node shows similar papillary configuration of metastatic carcinoma (hematoxylin and eosin $\times 100$ ).

biopsy, chest X-ray, Computed Tomography (CT) of the chest and abdomen, Magnetic Resonance Imaging (MRI) of the brain and abdomen, and bone scan. A chest X-ray and CT of the lung revealed a noncalcified right upper lobe lung mass. A bone scan showed widespread metastatic skeletal disease. MRI of the brain and abdomen revealed metastatic disease to the basal ganglia, thalamus, and liver. The patient had radiation therapy for brain metastasis but subsequently expired secondary to complications from his metastatic disease.

Pathology On gross examination of the right eye, a total retinal detachment was evident with subretinal hemorrhagic exudate. Underlying the temporal retina, a $19 \mathrm{~mm} \times 16 \mathrm{~mm} \times 12 \mathrm{~mm}$ grayish-white choroidal mass was evident. The tumor had a soft consistency and had focal areas of brown-red pigmentation within. On microscopic examination, the mass was noted to be a papillary tumor of epithelial cells with focal glandular structures surrounding a central fibrovascular core (Figure 1b). Tumor cells were polygonal in shape with eosinophilic cytoplasm and large nuclei with prominent nucleoli. Extracellular cystic spaces contained material which stained positive for PAS, mucicarmine, and alcian blue. The tumor was positive for cytokeratin, but negative for S-100, thyroglobulin, vimentin, and prostate-specific antigen. The supraclavicular lymph node biopsy was also noted to contain metastatic carcinoma in a similar papillary configuration (Figure 1c).

\section{Case 2}

A 53-year-old African-American man presented to the eye clinic with a 3-week history of distorted and blurred vision in both eyes, the left greater than the right, with associated left retro-orbital pain. He also complained of malaise and weight loss over the past 3 months. His past medical history was significant for a 5-year history of HIV without any acquired immunodeficiency syndrome (AIDS) defining illnesses. 
He was also diagnosed and treated for tuberculosis 15 years earlier. A history of hepatitis $\mathrm{C}$ and depression was also noted. His medications included lamivudine/zidovudine, indinavir sulfate, and sertraline hydrochloride. He had an extensive smoking history of 1-2 packs per day, and a history of alcohol and intravenous drug abuse.

On examination, visual acuity was $20 / 40$ in his right eye and 20/200 in his left eye. There was no afferent pupillary defect. Extraocular motility, confrontation field, and slitlamp examination were normal. There was a creamy colored mass with overlying striae at the posterior pole in both eyes, larger in the left eye. The appearance was suggestive of an exudative retinal detachment.

The differential diagnosis included posterior scleritis, tuberculosis, syphilis, sarcoid, Harada's disease, and metastatic cancer. A fluorescein angiogram was performed which revealed early hypofluorescence and patchy filling in both eyes with late pinpoint hyperfluorescence. A B-scan ultrasonography revealed choroidal solid lesions in the posterior pole, larger in the left eye. Adjacent subretinal fluid was also noted in both eyes.

A systemic evaluation included: complete blood count, FTA-ABS, angiotensin converting enzyme, lysosyme, ANA, sedimentation rate, and cryptococcal antigen, all of which were normal. The CD4 count was 810 and HIV RNA was 8254; based on this high CD4 count and low viral load, an AIDS-related infectious illness was considered unlikely. A chest X-ray revealed perihilar adenopathy suggestive of sarcoidosis. CT and MRI (Figure 2a) of the brain and orbits was normal except for evidence of an abnormal density in the posterior poles of both eyes that was not consistent with a mass lesion.

The patient was admitted to the hospital for further evaluation and treatment. Suspecting the posterior pole lesions were inflammatory in nature he was treated with systemic prednisone. In the first 2 days of treatment his vision improved; however, he developed pancreatitis and disseminated intravascular coagulation. Computed tomography of the abdomen and pelvis revealed a liver mass and lytic lesions to the bone. A bone marrow biopsy revealed carcinoma of unknown primary. The patient's hospital course rapidly deteriorated and he died before a primary was found. An autopsy was performed.

Pathology Gross examination of both eyes revealed areas of choroidal thickening. On microscopic examination, choroidal masses were noted bilaterally consisting of poorly differentiated epithelial cells in a pseudoglandular pattern consistent with metastatic carcinoma (Figure $2 b$ ). The remaining autopsy findings were significant for the presence of anaplastic large cell carcinoma of the lung, cardiac ventricles, pancreas, liver, and bone marrow. Although a definitive location for the primary focus of the carcinoma was never determined, the pulmonary focus was believed to be the most likely source (Figure 2c).

\section{Discussion}

A half-century ago, metastatic intraocular tumors were considered rare. ${ }^{5,6}$ Now, however, metastatic tumors are considered the most common intraocular tumors of adults. ${ }^{1}$ Nelson ${ }^{7}$ estimated that the total incidence of ocular metastases in patients with cancer is approximately $4 \%$. The apparent increased incidence of metastatic intraocular tumors is due to the longer survival of cancer patients from chemotherapy and radiation treatments as well as improved clinical detection of metastatic tumors by ophthalmologists. ${ }^{8}$

In a clinical survey of 520 eyes with uveal metastases at Wills Eye Hospital, primary tumors from breast and lung accounted for the majority of cases with lung being the most common site of origin in men (40\%). Of interest, at the time of ocular diagnosis, only $66 \%$ had a known primary tumor. In those without known cancer, a primary was found $49 \%$ of the time after subsequent investigation, with lung being the most common. ${ }^{2}$

The incidence of AIDS-related illness and mortality from AIDS have declined significantly due to the new drug therapies introduced in the last few years. Accordingly, HIV-related malignancies, ie, Kaposi sarcoma and lymphoma are also declining. ${ }^{9}$ However, other non-AIDS defining tumors, such as lung cancer, have increased in frequency in patients with HIV. ${ }^{3,4}$ Recent studies have suggested that lung cancers are more aggressive in HIV patients; it usually occurs at a younger age, and there is a shorter survival time.,

Why are there not then additional case reports of metastatic uveal carcinoma in HIV infection in the literature? One hypothesis may be that in the past, with poor survival, the overall prevalence of metastatic cancer in HIV patients was low; therefore, the prevalence of uveal metastases was also low. An alternative hypothesis is that these cases were misdiagnosed as, or masked by, concurrent ocular infectious disease. As is often the case, blurred vision was the first presenting sign of uveal metastases in these two patients, symptoms which were ignored for weeks (Case 2) and months (Case 1), respectively. In their series, Shields and colleagues ${ }^{2}$ noted that visual acuity in patients with uveal metastases was 20/60 or better in $40 \%$ of cases at time of diagnosis. Pain was 

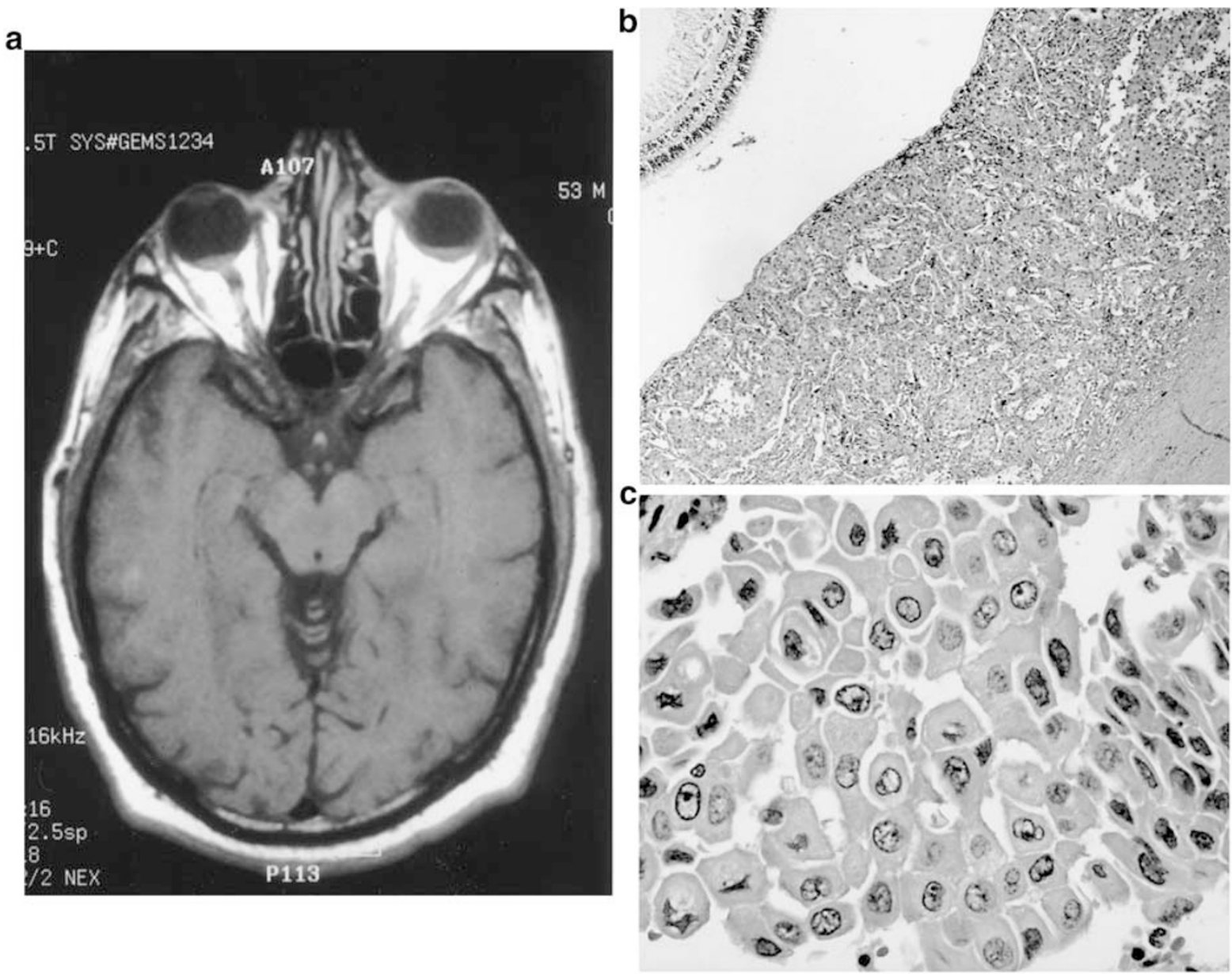

Figure 2 Case 2. (a) Magnetic resonance imaging demonstrates bilateral subretinal masses. (b) Histologic section from eye shows pseudoglandular pattern of sub-retinal metastatic carcinoma (hematoxylin and eosin $\times 100$ ). (c) Histologic section from lung shows atypical, pleomorphic, hyperchromatic epithelial cells (hematoxylin and eosin $\times 400$ ).

reported in only $7 \%$ of cases. This lack of significant symptomatology in patients may also contribute to the low reported incidence of uveal metastases because they are less inclined to undergo a detailed ocular exam.

One could speculate that, with the improved treatments for HIV and the longer survival times of infected individuals, uveal metastases from primary carcinomas will become more common. These cases illustrate that uveal metastatic disease should be considered in the differential diagnosis of HIV-infected patients who present to the ophthalmologist.

\section{Acknowledgements}

Supported in part by The Richard A Perritt Charitable Foundation. The authors have no proprietary or financial interest in any products used in this study.

\section{References}

1 Ferry AP, Font RL. Carcinoma metastatic to the eye and orbit: I. A clinico-pathologic study of 227 cases. Arch Ophthalmol 1974; 92: 276-286.

2 Shields CL, Shields JA, Gross NE, Schwartz GP, Lally SE. Survey of 520 eyes with uveal metastases. Ophthalmology 1997; 104: 1265-1276.

3 Alshafie MT, Donaldson B, Oluwole SF. Human immunodeficiency virus and lung cancer. Br J Surg 1997; 84: 1068-1071.

4 Parker MS, Leveno DM, Campbell TJ,Worrell JA, Carozza SE. AIDS-related brochogenic carcinoma: fact or fiction? Chest 1998; 113: 154-161.

5 Cordes FC. Bilateral metastatic carcinoma of the choroid with x-ray therapy to one eye: report of a case. Am J Ophthalmol 1944; 27: 1355-1370.

6 Greear JN. Metastatic carcinoma of the eye. Am J Ophthalmol 1950; 33: 1015-1025.

7 Nelson CC, Hertzberg BS, Klintworth GK. A histopathological study of 716 unselected eyes in patients with cancer at the time of death. Am J Ophthalmol 1983; 95: 788-793. 
8 Stephens RF, Shields JA. Diagnosis and management of cancer metastatic to the uvea: a study of 70 cases. Ophthalmology 1979; 86: 1336-1349.

9 Tirelli U, Spina M, Sandri S, Serraino D, Gobitti C, Fasan
M et al. Lung carcinoma in 36 patients with human immunodeficiency virus infection. The Italian Cooperative Group on AIDS and Tumors. Cancer 2000; 88: 563-569. 